A large aperture aplanatic lens not corrected for colour

This content has been downloaded from IOPscience. Please scroll down to see the full text. 1922 Trans. Opt. Soc. 2422

(http://iopscience.iop.org/1475-4878/24/1/304)

View the table of contents for this issue, or go to the journal homepage for more

Download details:

IP Address: 139.184.14.159

This content was downloaded on 01/10/2015 at 06:56

Please note that terms and conditions apply. 


\title{
A LARGE APERTURE APLANATIC LENS NOT CORRECTED FOR COLOUR
}

\author{
By T. SMITH, M.A., F.Inst.P. \\ (Optics Department, National Physical Laboratory)
}

$M S$. received, ist December, I922. Read and discussed, 14th December, 1922.

$A B S T R A C T$. A lens suitable for spectroscopic work with aplanatic corrections for all zones may have as large an aperture as $f / \mathrm{I}$ or still greater, all the surfaces being strictly spherical. An actual lens made by Messrs Ross, Ltd, of 3 inches focal length and 3 inches aperture, possesses corrections comparable with those given by the theoretical investigation. With a slightly reduced aperture, correction for colour may be obtained without prejudice to the quality of the spherical corrections. The investigation shows that the production of suitable glass discs is the outstanding difficulty in the way of great increases in the relative apertures of telescope objectives.

Although a single lens with spherical surfaces cannot form a real image of a real object free from spherical aberration, it is well known that if several such lenses of the same glass are placed in contact to form a system resembling that shown in Fig. I the removal of this aberration is possible*. Lenses of this type appear to have been regarded as a mere academic illustration of the principle of division to which the lens designer is occasionally compelled to resort, and so far as is known have not been employed for any purpose. The object of this note is to call attention to the remarkably fine correction of the central errors which may be achieved by their aid, and to the value lenses of this type possess for laboratory purposes where a very large relative aperture is of predominant im-

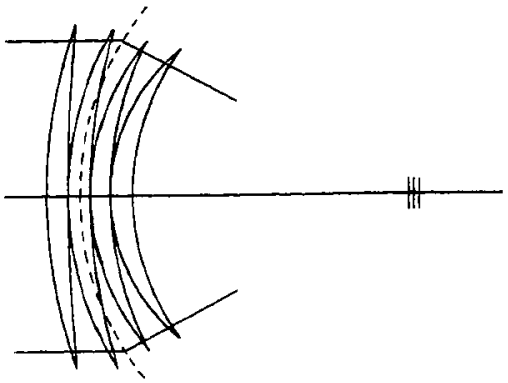

Fig. I portance, and the absence of correction for colour is of little, if any, consequence.

As in the lenses to be considered the diameter of the aperture may be as large as or even greater than the focal length, it is quite essential that they should be corrected for coma as well as for spherical aberration if image points off the axis are to be utilised. To determine the correct form for such lenses the two first order - conditions corresponding to the elimination of spherical aberration and of coma for a thin system composed of a number of thin lenses of the same kind of glass must therefore be considered $\dagger$. Suppose there are $n$ components, each of refractive

* See for instance von Rohr, The formation of images in optical instruments, p. 237.

+ For a proof of the expressions used in the following theoretical discussion reference may be made to the article on "Optical Calculations" in the Dictionary of Applied Physics, 4. 
index $\mu$, and that the $p$ th lens is of power $\kappa_{p}$ and that the sum of the curvatures of its surfaces is $2 \rho_{p}$. Then using the double suffix to denote the limits of summation, but omitting a suffix where the summation extends over the whole system, the difference between the curvatures of the unit surfaces is $\varpi \kappa$ where

$$
\pi=\frac{I}{\mu},
$$

the sum of the curvatures of the unit surfaces is $\beta_{k}$ where

$$
\beta \kappa^{2}=2(I+\varpi) \Sigma \rho_{p} \kappa_{p},
$$

and the spherical aberration for magnification $-I$ is proportional to $\gamma / \kappa$ where

$$
\begin{aligned}
\gamma \kappa^{3}=\Sigma\left\{(\mathrm{I}-\varpi)^{-2} \kappa_{p}^{2}+4(\mathrm{I}+2 \varpi) \rho_{p}{ }^{2}-8(\mathrm{I}+\varpi) \rho_{p}\left(\kappa_{1, p}-\kappa_{p, n}\right)\right. & \left.+(3+2 \varpi)\left(\kappa_{1, p}-\kappa_{p, n}\right)^{2}\right\} \kappa_{p} .
\end{aligned}
$$

For magnification $m$ the condition for the absence of spherical aberration is

$$
\gamma-4 \beta M+(3+2 \varpi) M^{2}=0
$$

and the condition for freedom from coma is

$$
\beta-(2+\varpi) M=0,
$$

where

$$
M=\frac{\mathrm{I}+m}{\mathrm{I}-m}
$$

If

$$
\sigma_{p}=(\mathrm{I}+2 \varpi) \rho_{p}-(\mathrm{I}+\varpi)\left(\kappa_{1, p}-\kappa_{p, n}+M \kappa\right) \text {. }
$$

these conditions may be rewritten

$$
\left(\frac{2+\varpi}{I-\varpi}\right)^{2} \Sigma_{\kappa_{p}}{ }^{3}+12 \Sigma \sigma_{p}{ }^{2} \kappa_{p}-\left(1+3 M^{2}\right) \kappa^{3}=0
$$

and

$$
2(\mathrm{I}+\varpi) \Sigma \sigma_{\nu} \kappa_{p}-\pi M \kappa^{2}=0
$$

When all the components are positive the first two terms in the spherical aberration condition are necessarily positive, and for real images $\left(M^{2} \ngtr \mathrm{r}\right)$ tend to exceed the only negative term when $n$ is small. The positive terms have their least value when the power of each component lens is equal and each $\sigma$ is zero. The second equation however shows that it is not permissible to make the $\sigma$ 's vanish, as too much coma is thereby introduced. The most favourable conditions are evidently secured by making all the $\sigma$ 's equal and satisfying (2). The rather more general value

$$
\sigma_{p}=\frac{\pi}{2(\mathrm{I}+\varpi)} M_{\kappa}+\alpha\left(\kappa_{1, p}-\kappa_{p, n}\right)
$$

satisfies (2) identically and gives for (I)

$$
\left(\begin{array}{l}
2+\varpi \\
1-\varpi
\end{array}\right)^{2}+4 a^{2}\left(n^{2}-1\right)+n^{2}\left\{3\left(\frac{M \varpi}{1+\pi}\right)^{2}-3 M^{2}-\mathrm{I}\right\}=0 \ldots(3) \text {. }
$$

All lenses to which this theory applies thus consist of a number of components of equal power in axial contact and separated by air spaces bounded by equal curvature differences, such as the lens shown in section in Fig. I.

If a system has been obtained with the desired correction for coma but overcorrected for spherical aberration, this fault can be remedied by giving $\alpha$ a suitable 
finite value. In the determination of illustrative systems the value zero will be assigned to $\alpha$ in the first place. It will be further assumed that the object is at infinity, so that $M=\mathrm{I}$. If the coma were neglected, the first term in the last bracket in (3) would be absent, and the spherical aberration could then be removed if

giving as particular cases

$$
\text { क } \ngtr \frac{2(n-I)}{2 n+I} \text { or } \mu \nLeftarrow \frac{2 n+I}{2\left(n-\frac{I}{I}\right)}
$$

$$
\begin{array}{lll}
n=2, & \varpi \ngtr \frac{2}{3}, & \mu \nless 2.5 \\
n=3, & \varpi \ngtr 4, & \mu \nless \mathrm{I} \cdot 75 \\
n=4, & \varpi \ngtr \frac{2}{3}, & \mu \nless \mathrm{I} \cdot 5
\end{array}
$$

agreeing with the values stated by von Rohr. When coma also is to be corrected the minimum refractive indices will be raised and for $n=4$, which the above figures suggest for the range of media available, it is found that the minimum value of $\varpi$ is very approximately $\cdot 648$, corresponding to a minimum index of $\mathrm{I} \cdot 5432 \mathrm{I}$. Using this value of $\varpi$, the curvatures given by the foregoing equations are, for unit focal length,

$$
\begin{array}{r}
\cdot 49518 \\
\cdot 03496 \\
\cdot 85407 \\
\cdot 39385 \\
\mathrm{I} \cdot 2 \mathrm{I} 295 \\
\cdot 75273 \\
\mathrm{I} \cdot 57 \mathrm{I} 84 \\
\mathrm{I} \cdot \mathrm{I} I \mathrm{I} 62
\end{array}
$$

If each lens is given an axial thickness equal to $\cdot 0625$, the aperture attainable will be approximately equal to the focal length. The results then obtained on tracing through rays at incident heights $0, \cdot 3, \cdot 4, \cdot 5$ are as follow:

$\begin{array}{ccc}\text { Incident height } & \begin{array}{c}\text { Intersection } \\ \text { distance }\end{array} & \text { Focal length } \\ 0 & .82796 & .97194 \\ \cdot 3 & .82992 & .97975 \\ \cdot 4 & .83014 & .98486 \\ \cdot 5 & .82887 & .99047\end{array}$

The spherical aberration plotted as curve $a$, Fig. 2, is somewhat overcorrected, but the variation in the focal length, shown as curve $a$ in Fig. 3, amounts to about 2 per cent., and this must be corrected by increasing the curvature of every surface by a constant amount. The first order formula indicates an addition of between .08 and 09 . Taking the former, and again tracing rays through leads to the results:

$\begin{array}{ccc}\text { Incident height } & \begin{array}{c}\text { Intersection } \\ \text { distance }\end{array} & \text { Focal length } \\ 0 & -81654 & 96616 \\ \cdot 4 & .81591 & .96812 \\ 5 & .81544 & .96912\end{array}$

The variation in the focal length, shown in curve $b$ of Fig. 3, is now only about $\frac{1}{3}$ per cent., but the change of curvature has transformed the spherical aberration, 
curve $b$ of Fig. 2, from the overcorrected to the undercorrected condition. There is no remedy for the latter but an increase of refractive index. Comparison of the results obtained with the two indices $I \cdot 5$ and $I \cdot 54321$ suggests $I \cdot 6$ as a suitable

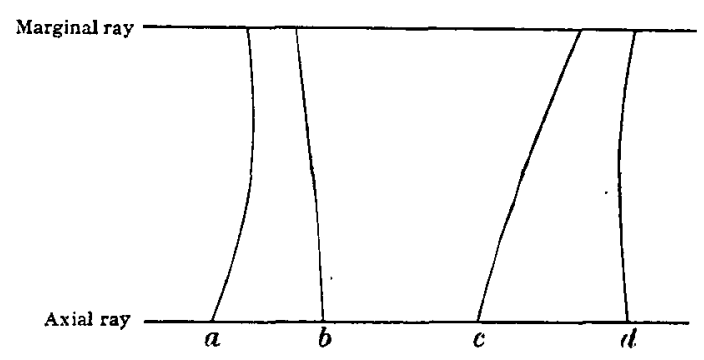

Fig. 2. Spherical Aberration

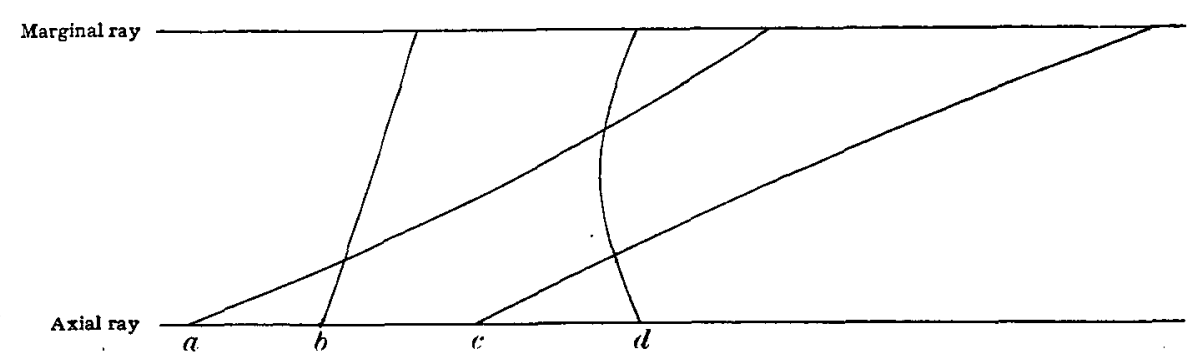

Fig. 3. Coma

total index, so that $\varpi=625$. Now solve with this index for no coma and minimum spherical aberration. The curvatures obtained are

$$
\begin{array}{r}
.47436 \\
.05769 \\
.83547 \\
\cdot 41880 \\
I \cdot 19658 \\
\cdot 77991 \\
I \cdot 55769 \\
I \cdot 14103
\end{array}
$$

Introducing the same thicknesses as before and tracing rays through gives:

$\begin{array}{ccc}\text { Incident height } & \begin{array}{c}\text { Intersection } \\ \text { distance }\end{array} & \text { Focal length } \\ 0 & .81847 & .963 \times 2 \\ \cdot 5 & .8215 \mathrm{r} & .9848 \mathrm{r}\end{array}$

The spherical aberration and coma curves for this lens are shown at $c$ in Figs. 2 and 3 respectively. For the removal of the coma the first order equation suggests 
a curvature addition of $I 15$. Making this correction and again tracing the rays gives:

$\begin{array}{ccc}\text { Incident height } & \begin{array}{c}\text { Intersection } \\ \text { distance }\end{array} & \text { Focal length } \\ 0 & .80032 & 95331 \\ -7 & 79994 & 95221 \\ -7 & .79991 & .95215 \\ -80029 & 95320\end{array}$

showing that the necessary index and curvature additions have been correctly estimated. The two aberration curves are marked $d$ in Figs. 2 and 3 .

It will be observed that these figures indicate a surprisingly small amount of spherical aberration-about $f / 2500$-in a lens corrected for an aperture of $f / .95$.

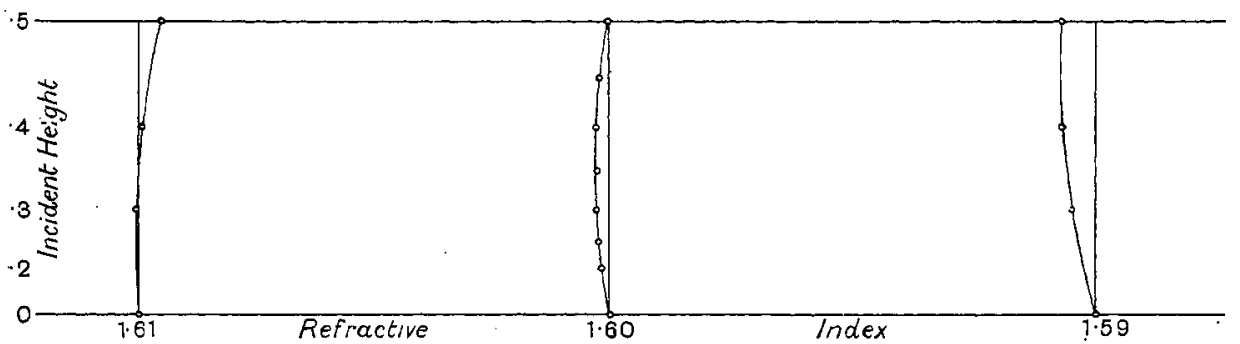

Fig. 4. Zonal variation of Spherical Aberration

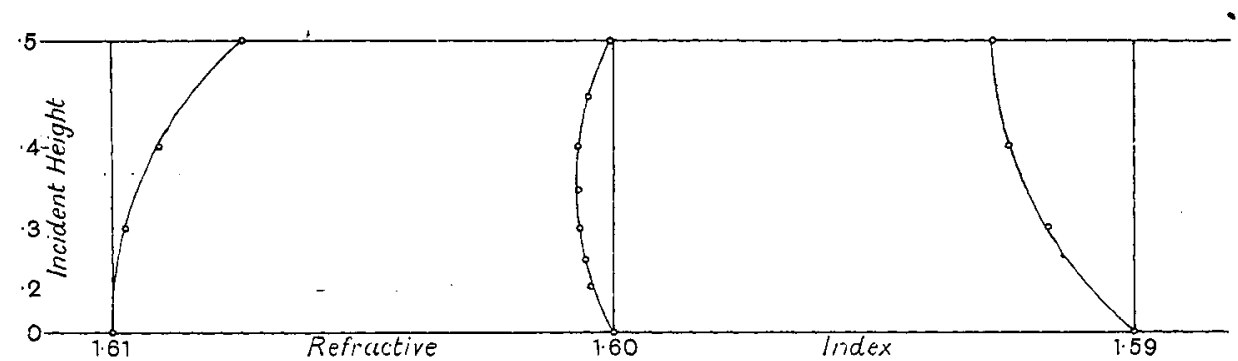

Fig. 5. Zonal variation of Coma

As a matter of interest other rays were traced and the resulting points are distinguished by small circles on the central curves in Figs. 4 and 5. The ordinates in both cases are the squares of the incident heights. The curves drawn through the points are circular arcs, and it is seen that the points lie satisfactorily on these curves. The position of focus determined experimentally should correspond closely with that of the vertical straight line drawn through the curve of Fig. 4 so as to intercept equal areas on either side between the straight line and the curve limited at the two ends by the axial and marginal heights. By making a slight change in the ordinates, and plotting on a uniform scale proportional to $\mathrm{I}-\cos \psi$ instead of $\sin ^{2} \psi$, the corresponding vertical straight line denotes the position of focus for which the axial and marginal rays agree in phase. It is easy from this figure to 
show that the path differences at the experimental focus will be approximately as stated in the following table:

\begin{tabular}{c|c|c|c} 
Incident height & $\begin{array}{c}\text { Intersection } \\
\text { distance from } \\
\text { mean focus } \times 10^{5}\end{array}$ & $\begin{array}{c}\text { Focal length } \\
\text { minus mean } \times 10^{5}\end{array}$ & $\begin{array}{c}\text { Optical path } \\
\text { minus mean } \times 10^{7}\end{array}$ \\
\hline 0 & 27 & 75 & 0 \\
-2 & -6 & 0 & -34 \\
-25 & -11 & -14 & -36 \\
3 & -9 & -35 & -27 \\
35 & -14 & -41 & -5 \\
4 & -3 & 6 & 36 \\
45 & 24 & 64 & 0 \\
\hline 5 & & & 22 \\
\hline
\end{tabular}

If light is used for which the refractive index differs from $\mathrm{I} \cdot 6$ the position of the focus will change rapidly as the lens is quite uncorrected for colour, and the character of the aberrations will alter. It is to be expected that the aberrations will change at a relatively slow rate, so that when the image for a different wave length is correctly focussed a good image will be obtained if the change in wave length is not too great. To form an estimate of the changes of aberration it is convenient to disregard the probable change of wave length and take arbitrary differences of refractive index. Rays have therefore been traced through the system drawn to scale in Fig. I, as it stands for the index I.60, viz.

\begin{tabular}{|c|c|}
\hline Curvatures & Axial thicknesses \\
\hline $\begin{array}{l}58936 \\
17269\end{array}$ & .0625 \\
\hline $\begin{array}{l}95047 \\
53380\end{array}$ & .0625 \\
\hline $\begin{array}{r}\text { I.3II58 } \\
.89491\end{array}$ & .0625 \\
\hline $\begin{array}{l}1 \cdot 67269 \\
1 \cdot 25603\end{array}$ & .0625 \\
\hline
\end{tabular}

when the index is given in turn the values $I \cdot 59$ and $I \cdot 6 I$. The results are

\begin{tabular}{|c|c|c|c|}
\hline $\begin{array}{l}\text { Refractive } \\
\text { index }\end{array}$ & $\begin{array}{c}\text { Incident } \\
\text { height }\end{array}$ & $\begin{array}{l}\text { Intersection } \\
\text { distance }\end{array}$ & $\begin{array}{l}\text { Focal } \\
\text { length }\end{array}$ \\
\hline 1.59 & $\begin{array}{l}0 \\
\cdot 3 \\
\cdot 4 \\
\cdot 5\end{array}$ & $\begin{array}{l}81585 \\
.81511 \\
.81480 \\
.81479\end{array}$ & $\begin{array}{l}96992 \\
.96724 \\
96596 \\
96544\end{array}$ \\
\hline 1.61 & $\begin{array}{l}0 \\
\cdot 3 \\
\cdot 4 \\
\cdot 5\end{array}$ & $\begin{array}{l}78532 \\
.78525 \\
.78544 \\
.78607\end{array}$ & $\begin{array}{r}93725 \\
.93765 \\
\cdot 93877 \\
\cdot 94148\end{array}$ \\
\hline
\end{tabular}

showing that reasonably good correction can be secured over, say, the whole extent of the visible spectrum. Figs, 4 and 5 show in their correct relative positions the aberration curves for these three refractive indices; the positions of the three 
corresponding paraxial focussing planes are marked in Fig. $x$. The variations of path will be roughly four times that for $I .6$ when the index is $I \cdot 59$, and three times when the index is $\mathrm{I} \cdot 6 \mathrm{I}$. On taking the variations of wave length into account, and measuring the variations at each focus in terms of the wave length of the light for which the image is formed there, the corrected figures will be approximately three times at 1.59 and four times the number of wave lengths at $\mathrm{I} \cdot 6 \mathrm{I}$. It should be evident from the diagram illustrating this lens that the limit to which the aperture can be pushed has by no means been reached when it is approximately equal to the focal length. The theoretical limit, which of course is quite unattainable, is reached when the diameter of the aperture is twice the focal length, for in that case if spherical aberration and coma are corrected the emergent marginal ray will be perpendicular to the axis. The area of the aperture in the case just considered is thus one-quarter of the maximum indicated by theory. By doubling the thickness of each lens and the curvature addition to the approximate solution the available area of aperture will be approximately doubled while the coma will remain roughly . corrected. It is to, be expected that the aberrations remaining in the system will be at least four times as great as those found with the smaller aperture, and that a further increase in index or in the number of the components will be necessary to secure correction for spherical aberration. Systems of this greater aperture have been investigated at this Laboratory by Miss Everett.

A lens to this design of three inches aperture and three inches focal length has been constructed for a special research by Messrs Ross, Ltd, who courteously permitted the author to see the instrument when it was completed. Examination in ordinary light is not possible on account of chromatic aberration, for with so large an angular aperture the image for any particular wave length is lost in the flood of light from other spectral regions. The method adopted was to use, at the focus of a large collimator, an artificial star consisting of a small steel ball illuminated by a mercury vapour lamp. The actual lens showed errors of the order of magnitude expected from the calculations. The out of focus images when both yellow and blue radiations were admitted were particularly interesting. The useful field of view is naturally small, but the angular field for which the correction can be considered acceptable is comparable with that given by an ordinary telescope objective of similar linear aperture. As the lens is corrected for both spherical aberration and coma the first aberration to make its appearance on leaving the axis is of the symmetrical type. Its outline resembles a flat loop, the central parts of which have been crossed.

It will be noted from Figs. 2 and 3 that for the lower index the aberration curves are concave to the lens, and those for the higher index are convex. This implies that curves indistinguishable from a straight line are theoretically possible, that is a lens of this aperture may be perfectly corrected. The attainment of this state of correction would probably follow from a more detailed investigation of lenses of this type in which the ratios of the higher order aberrations arising at each surface to those of lower orders were suitably controlled by modifications in the lens curvatures. In this connection it may not be uninteresting to give the 
amounts of the aberrations arising at each surface in the final lens, taking as the measure of the aberration the excess of the length of the perpendicular from the vertex to the refracted marginal ray over that to the incident marginal ray. The values are given in the following table:

\begin{tabular}{c|c|c}
\hline Surface & $\begin{array}{c}\text { First order } \\
\text { aberration } \times 10^{5}\end{array}$ & $\begin{array}{c}\text { Total aberration of } \\
\text { all orders } \times 10^{5}\end{array}$ \\
\hline I & 509 & 532 \\
2 & -65 & -65 \\
3 & 269 & -287 \\
4 & -11 & -12 \\
5 & -104 & -13 \\
6 & -524 & -579 \\
7 & 11 & I 2 \\
\hline
\end{tabular}

The great difference between a lens of large aperture and one of ordinary properties is hardly appreciated when expressed in the ordinary relative aperture notation. Compared with an ordinary telescope objective of the same aperture and with spherical errors of the same order of magnitude the present lens will yield an image about 200 times brighter. If the aberration curves of an ordinary objective were plotted on the same scale as the curves of Figs. 2 to 5 their total heights would be about one-quarter the diameter of one of the circles indicating the positions of the calculated points on the two latter diagrams. As the relative apertures of objectives of the usual design are limited by the appearance of aberrations which can only be controlled by the use of non-spherical surfaces, it will be seen that objectives of the new type offer corrections of a quite distinct order of accuracy from any attainable with lenses of normal design.

The objective as described differs from the ordinary lens in being uncorrected for colour. This feature is evidently not inherent in lenses of the new type. With a refractive index of about 1.6 there are available glasses of small dispersion, such as the dense barium crowns, and also dense flints in which the dispersion is large. By replacing each single component of the lens that has been described by an achromatic combination of such glasses having the same index for light of a selected wave length it is evident that chromatic correction can be attained at the cost of approximately halving the rapidity. Thus if the glass discs were available large telescope objectives could be constructed with a rapidity for extended sources of the order of 100 times that of existing instruments. It is by no means necessarily the case that a construction of this type is the best that can be obtained in an achromatic objective with eight glass discs, but it is of interest as evidence that if the problems connected with the production of the glass can be surmounted the theoretical difficulties of designing a telesçope of this new order are not likely to prove a serious obstacle in the way of its realisation.

In conclusion the author desires to express his thanks to Miss Everett and Mr F. J. C. Brookes for their assistance in this investigation. 


\section{DISCUSSION}

Dr L. C. Martin: Could the correction not have been carried out in a somewhat similar way to that used in the design of microscope objectives?

Mr Smith: In the case of a microscope objective one is dealing with a system which is small in actual dimensions. As the path differences are proportional to the dimensions, it is not likely that one could increase the scale of such a system unless its path differences were very nearly zero. In the present lens the total variation in path is less than one wave length over the whole aperture. 